\title{
Infinite dimensional and reduced order observers for Burgers equation
}

\author{
M. Ö. Efe , H. Özbay \& M. Samimy
}

To cite this article: M. Ö. Efe , H. Özbay \& M. Samimy (2005) Infinite dimensional and reduced order observers for Burgers equation, International Journal of Control, 78:11, 864-874, DOI: $10.1080 / 00207170500158813$

To link to this article: http://dx.doi.org/10.1080/00207170500158813

$$
\text { 曲 Published online: } 20 \text { Feb } 2007 .
$$

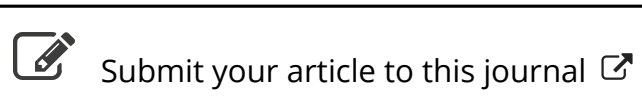

Џll Article views: 57

Q View related articles $\asymp$

4 Citing articles: 4 View citing articles 


\title{
Infinite dimensional and reduced order observers for Burgers equation
}

\author{
M. Ö. EFE*†, H. ÖZBAY† and M. SAMIMY§ \\ $\dagger$ TOBB University of Economics and Technology, Department of Electrical and Electronics Engineering, \\ TR-06530, Ankara, Turkey \\ $\$$ Department of Electrical and Electronics Engineering, Bilkent University, Bilkent, TR-06800, Ankara, Turkey \\ §Department of Mechanical Engineering, The Ohio State University, Columbus, OH 43210, USA
}

(Received August 2004; revised March 2005; in final form May 2004)

\begin{abstract}
Obtaining a representative model in feedback control system design problems is a key step and is generally a challenge. For spatially continuous systems, it becomes more difficult as the dynamics is infinite dimensional and the well known techniques of systems and control engineering are difficult to apply directly. In this paper, observer design is reported for one-dimensional Burgers equation, which is a non-linear partial differential equation. An infinite dimensional form of the observer is demonstrated to converge asymptotically to the target dynamics, and proper orthogonal decomposition is used to obtain the reduced order observer. When this is done, the corresponding observer is shown to be successful under certain circumstances. The paper unfolds the connections between target dynamics, observer and their finite dimensional counterparts. A set of simulation results has been presented to justify the theoretical claims of the paper.
\end{abstract}

\section{Introduction}

Investigating the behaviour of systems having many states is a core research field particularly in spatially continuous systems. Without loss of generality, a system characterized by Partial Differential Equations (PDE) is a good example. In such cases, typically the modelling approach assumes that the response of the system is dominated by coherent modes and the task is to get a useful dynamical model containing only the significantly energetic modes. From this point of view, one can obtain a reduced order model for an infinite dimensional system. The question is whether this model can be used for constructing a state information. The approach adopted in this paper demonstrates that it is possible for Burgers equation, which captures essential features of the Navier-Stokes equations. In fact one can argue that any modelling and control technique aimed at flow problems described by the Navier-Stokes equations has to work for the Burgers equation. In other words, Burgers

*Corresponding author. Email: onderefe@ieee.org equation is a good starting point to deal with more general flow problems. What make the problem challenging are the infinite dimensionality and non-linearity, and due to these reasons, the classical design approaches of the systems and control theory are difficult to apply directly. Burgers equation has previously been considered for model reduction and controller design purposes (Krstić 1999, Liu and Krstić 2000, Vedantham 2000, Liu and Krstić 2001, Burns et al. 2002a, b, Hinze and Volkwein 2002, Park and Jang 2002, Efe and Özbay 2003a). This paper approaches the observer design problem from a control specialist's point of view, i.e. a suitable low dimensional modelling followed by a stability analysis in terms of Lyapunov theory. From this point of view, the contribution of this paper is that a low dimensional observer could be used to estimate the most significant temporal information in the dynamics of infinite dimensional Burgers equation. Furthermore, the approach stipulates that a similar scheme could be extended to higher-dimensional PDEs.

Design of an observer for Burgers equation contains three major issues that need to be studied carefully. The first issue is the modelling, i.e. collecting the 
representative data and exploiting several techniques to come up with a set of Ordinary Differential Equations (ODEs). The second issue is to separate the effect of external stimuli from the other terms by using the boundary conditions. The third issue is to design an observer that estimates the most dominant states. The process is continuous over a physical domain $(\Omega)$, the boundaries of which are the possible entries of external stimuli. Choosing an adequately dense grid, say $\Omega_{d}$, lets us obtain a finite size representation of the process $u(x, t)$ over $\Omega_{d}$. When the content of the observed data, say $u(x, t)$, is decomposed into spatial and temporal constituents $\left(u(x, t) \approx\langle\Phi(x), \alpha(t)\rangle_{\Omega_{d}}\right)$, the essence of spatial behaviour appears as a set of spatially varying gains $\left(\Phi(x)=\left\{\Phi_{1}(x), \Phi_{2}(x), \ldots, \Phi_{R_{L}}(x)\right\}\right)$, and the essence of temporal evolution, $\alpha(\mathrm{t})$, appears as the solution of a set of ODEs obtained after utilizing the orthogonality properties of the spatial basis functions. Having this picture in front of us, the goal is to construct the temporal evolution in the state space form. Needless to say, the spatial gains will appear in the output equation.

When the low dimensional modelling issue is taken into consideration, Proper Orthogonal Decomposition (POD), or Singular Value Decomposition (SVD) in cooperation with Galerkin projection are the popular approaches utilized frequently in the literature (Ravindran 2000, Ly and Tran 2001, Singh et al. 2001, Efe and Özbay 2003a, b, 2004 and the references therein). In Gügercin and Antoulas (2000), a good comparison of model reduction techniques is presented. The decomposition based methods use a library of solutions obtained from the process, and separate the content of the data in such a way that the spatial components (basis functions) display certain orthogonality properties and the temporal components synthesize the time evolution over those spatial basis functions. The decomposition yields meaningful information as long as the data contains coherent modes. One has to know that the result of POD or SVD schemes will be a set of basis functions accompanied by a set of autonomous ODEs.

The next issue, which is the separation of boundary condition(s) (or the control input(s)) from the remaining terms, is a key step. For example Krstić describes a neatly selected Lyapunov function in Krstic (1999), and the expression in its time derivative enables us to apply integration by parts, then the boundary condition emerges in an explicit manner. Although the approach lets the designer manipulate Dirichlét and Neumann type boundary conditions on Burgers equation, it is still tedious to follow the same procedure for more complicated PDEs. This can be because of the high dimensionality of the PDE in particular, and difficulty in finding an appropriate Lyapunov function in general. Therefore, utilizing the numerical techniques is a practical alternative to describe reduced order models for complicated systems of PDEs. A key contribution of this paper is to explain how the issue of control separation is handled in numerical data based modelling approaches.

A quick look at the literature on Burgers equation unfolds the fact that the majority of the works focuses on control system design. For example, receding horizon optimal control approach in Hinze and Volkwein (2002), stabilization by feedback control in Burns et al. (2002a, b), optimal control design in Vedantham (2000), iterative methods in Park and Jang (2002), adaptive control in Liu and Krstić (2001), backstepping control in Liu and Krstić (2000), Lyapunov mehods for stabilization in Krstić (1999), simple schemes like linearization based integral control in Efe and Özbay (2003a), and so on. A common property in these works is that the design is based on input-output information. It now becomes evident that a finite dimensional observer would make the state-space techniques applicable, which is the topic of discussion in this paper.

The paper is organized as follows: $\S 2$ introduces the infinite dimensional form of the observer. Section 3 briefly presents the POD technique and its relevance to the modelling strategy. In the $\S 4$, development of the reduced order model for the Burgers equation is analyzed and a typical situation is studied. The connections between the reduced order models and full order models are studied in $\S 5$, and a simulation study is described in $\S 6$. The relationship between the techniques presented in the paper and flow modelling and control are briefly discussed in $\S 7$. Finally, concluding remarks are made in $\S 8$.

\section{An infinite dimensional observer}

Consider the Burgers equation $u_{t}=\epsilon u_{x x}-u u_{x}$, where $\epsilon$ is a known constant, and the subscripts ${ }_{x}$ and ${ }_{t}$ refer to the partial differentiation with respect to $x$ and time, respectively. The spatial domain of the process is $\Omega:=\{x \mid x \in[0,1]\}$. The initial conditions are $u(x, 0)=$ $0 \forall x \in \Omega$, and the boundary conditions are $u(0, t)=0$ and $u(1, t)=\gamma(t)$, which is the free boundary input (boundary condition, or the control input). Similarly, for the observer, $v(x, t)$, we will have $|v(x, 0)|<$ $\infty \forall x \in \Omega, v(0, t)=0$ and $v(1, t)=\gamma(t)$.

Theorem 1: For the Burgers equation, an infinite dimensional observer having the structure given by

$$
v_{t}=\epsilon v_{x x}-v v_{x}+K(u-v)
$$

with any positive $K \in \Re$ results in globally exponentially stable reconstruction over $(x, t) \in[0,1] \times[0, \infty)$. 
Proof: Choose the following Lyapunov function candidate

$$
\begin{gathered}
V_{o}=\frac{1}{2} \int_{\Omega}(u-v)^{2} \mathrm{~d} x=\frac{1}{2} \int_{0}^{1}(u-v)^{2} \mathrm{~d} x \\
\dot{V}_{o}=\int_{0}^{1}(u-v)(\dot{u}-\dot{v}) \mathrm{d} x \\
=\int_{0}^{1}(u-v)\left(\epsilon u_{x x}-u u_{x}-\epsilon v_{x x}+v v_{x}-K(u-v)\right) \mathrm{d} x \\
=-2 K V_{o}+\int_{0}^{1}\left(\epsilon u u_{x x}-u^{2} u_{x}\right) \mathrm{d} x+\int_{0}^{1}\left(\epsilon v v_{x x}-v^{2} v_{x}\right) \mathrm{d} x \\
-\int_{0}^{1}\left(\epsilon v u_{x x}-v u u_{x}\right) \mathrm{d} x-\int_{0}^{1}\left(\epsilon u v_{x x}-u v v_{x}\right) \mathrm{d} x
\end{gathered}
$$

Since $\int_{0}^{1} \epsilon u u_{x x} \mathrm{~d} x=\epsilon \gamma u_{x}(1, t)-\epsilon \int_{0}^{1} u_{x}^{2} \mathrm{~d} x$, we have

$$
\begin{aligned}
\dot{V}_{o}= & -2 K V_{o}+\epsilon \gamma\left(u_{x}(1, t)+v_{x}(1, t)\right)-\epsilon \int_{0}^{1}\left(u_{x}^{2}+v_{x}^{2}\right) \mathrm{d} x \\
& -\int_{0}^{1}\left(\epsilon v u_{x x}-v u u_{x}\right) \mathrm{d} x-\int_{0}^{1}\left(\epsilon u v_{x x}-u v v_{x}\right) \mathrm{d} x \\
& -\int_{0}^{1}\left(u^{2} u_{x}+v^{2} v_{x}\right) \mathrm{d} x
\end{aligned}
$$

It is apparent that $\int_{0}^{1} \epsilon v u_{x x} \mathrm{~d} x=\epsilon \gamma u_{x}(1, t)-\epsilon \int_{0}^{1} u_{x} v_{x} \mathrm{~d} x$. This lets us have the equality

$$
\begin{aligned}
-\int_{0}^{1} \epsilon\left(v u_{x x}+u v_{x x}\right) \mathrm{d} x= & -\epsilon \gamma\left(u_{x}(1, t)+v_{x}(1, t)\right) \\
& +2 \epsilon \int_{0}^{1} u_{x} v_{x} \mathrm{~d} x
\end{aligned}
$$

and by the use of this relation, (4) can be rewritten as follows:

$$
\begin{aligned}
\dot{V}_{o}= & -2 K V_{o}-\epsilon \int_{0}^{1}\left(u_{x}-v_{x}\right)^{2} \mathrm{~d} x+\int_{0}^{1}\left(u v v_{x}+v u u_{x}\right) \mathrm{d} x \\
& -\int_{0}^{1}\left(u^{2} u_{x}+v^{2} v_{x}\right) \mathrm{d} x .
\end{aligned}
$$

Rearranging (6) yields

$$
\begin{aligned}
\dot{V}_{o}=- & 2 K V_{o}-\epsilon \int_{0}^{1}\left(u_{x}-v_{x}\right)^{2} \mathrm{~d} x+\int_{0}^{1}(u-v)\left(u u_{x}-v v_{x}\right) \mathrm{d} x \\
=- & 2 K V_{o}-\epsilon \int_{0}^{1}\left(u_{x}-v_{x}\right)^{2} \mathrm{~d} x+\int_{0}^{1}(u-v) \\
& \times\left(\frac{1}{2} \frac{\partial}{\partial x}\left(u^{2}-v^{2}\right)\right) \mathrm{d} x
\end{aligned}
$$

Set $\bar{\delta}=u^{2}-v^{2} \quad$ and note that $\bar{\delta}(0, t)=u^{2}(0, t)-$ $v^{2}(0, t)=0$ and $\bar{\delta}(1, t)=u^{2}(1, t)-v^{2}(1, t)=\gamma^{2}-\gamma^{2}=0$.
Defining $\Upsilon(\bar{\delta}, u, v)=(u-v)(1 / 2)(\partial \bar{\delta} / \partial x)$, and using the above identities we have the following result

$$
\begin{aligned}
\dot{V}_{o} & =-2 K V_{o}-\epsilon \int_{0}^{1}\left(u_{x}-v_{x}\right)^{2} \mathrm{~d} x+\int_{\bar{\delta}(0, t)=0}^{\bar{\delta}(1, t)=0} \Upsilon(\bar{\delta}, u, v) \mathrm{d} \bar{\delta} \\
& =-2 K V_{o}-\epsilon \int_{0}^{1}\left(u_{x}-v_{x}\right)^{2} \mathrm{~d} x<0 .
\end{aligned}
$$

According to the above result, regardless of the difference between $u(x, 0)$ and $v(x, 0)$, the integral of the square of $u(x, t)-v(x, t)$ converges asymptotically to zero. This clearly forces $v(x, t) \rightarrow u(x, t)$ exponentially as $t \rightarrow \infty$. It is worth emphasizing that the key technical assumption is the match at the boundary, i.e. $u(0, t)=v(0, t)$ and $u(1, t)=v(1, t)$. So, the knowledge of the boundary values is sufficient to design an infinite dimensional observer.

\section{Proper orthogonal decomposition}

Consider the ensemble $U_{i}(x), i=1,2, \ldots, N_{s}$, where $N_{s}$ is the number of elements. Every element of this set corresponds to a snapshot observed from a process, say for example, one-dimensional Burgers equation

$$
u_{t}(x, t)=\epsilon u_{x x}(x, t)-u(x, t) u_{x}(x, t) .
$$

The continuous time process takes place over the physical domain $\Omega:=\{x \mid x \in[0,1]\}$ and the solution is obtained on a grid denoted by $\Omega_{d}$, which describes the coordinates of the pixels of every snapshot in the ensemble.

The goal is to find an orthonormal basis set letting us to write the solution as

$$
u(x, t) \approx \hat{u}(x, t)=\sum_{i=1}^{R_{L}} \alpha_{i}(t) \Phi_{i}(x)
$$

where $\alpha_{i}(t)$ is the temporal part, $\Phi_{i}(x)$ is the spatial part, $\hat{u}(x, t)$ is the finite element approximate of the infinite dimensional PDE and $R_{L}$ is the number of independent basis functions that can be synthesized from the given ensemble, or equivalently that spans the space described by the ensemble. It will later be clear that if the basis set $\left\{\Phi_{i}(x)\right\}_{i=1}^{R_{L}}$ is an orthonormal set, then the modelling task can exploit Galerkin projection technique.

Let us summarize the POD procedure.

Step 1: Start calculating the $N_{s} \times N_{s}$ dimensional correlation matrix $L$, the (ij)th entry of which is 
$L_{i j}:=\left\langle U_{i}, U_{j}\right\rangle_{\Omega_{d}}$, where $\langle\cdot, \cdot\rangle_{\Omega_{d}}$ is the inner product operator defined over the chosen spatial grid $\Omega_{d}$.

Step 2: Find the eigenvectors (denoted by $w_{i}$ ) and the associated eigenvalues $\left(\lambda_{i}\right)$ of the matrix $L$. Sort them in a descending order in terms of the magnitudes of $\lambda_{i}$. Note that every $w_{i}$ is an $N_{s} \times 1$ dimensional vector satisfying $w_{i}^{\mathrm{T}} w_{i}=1 / \lambda_{i}$. For simplicity of the exposition, we assume here that the eigenvalues are distinct.

Step 3: Construct the basis set by using

$$
\Phi_{i}(x)=\sum_{j=1}^{N_{s}} w_{i j} U_{j}(x)
$$

where $w_{i j}$ is the $j$ th entry of the eigenvector $w_{i}$, and $i=1,2, \ldots, R_{L}$, where $R_{L}=\operatorname{rank}(L)$. It can be shown that $\left\langle\Phi_{i}(x), \Phi_{j}(x)\right\rangle_{\Omega_{d}}=\delta_{i j}$ with $\delta_{i j}$ being the Kronecker delta function. Notice that the basis functions are admixtures of the snapshots (Ly and Tran 2001, Efe and Özbay 2003b, 2004).

Step 4: Calculate the temporal coefficients. Taking the inner product of both sides of $(10)$ with $\Phi_{i}(x)$, the orthonormality property leads to

$$
\alpha_{i}\left(t_{0}\right)=\left\langle\Phi_{i}(x), \hat{u}\left(x, t_{0}\right)\right\rangle_{\Omega_{d}} \approx\left\langle\Phi_{i}(x), U_{t_{0}}\right\rangle_{\Omega_{d}} .
$$

Without loss of generality, an element of the ensemble $\left\{U_{i}(x)\right\}_{i=1}^{N_{s}}$ may be $U\left(x, t_{0}\right)$. Therefore, in order to generate the temporal gain, $\alpha_{k}(t)$, of the spatial basis $\Phi_{k}(x)$, one would take the inner product with the elements of the ensemble with the basis functions as given below.

$$
\begin{aligned}
\left\langle U_{1}(x), \Phi_{k}(x)\right\rangle_{\Omega_{d}} & \approx \alpha_{k}\left(t_{1}\right) \\
\left\langle U_{2}(x), \Phi_{k}(x)\right\rangle_{\Omega_{d}} & \approx \alpha_{k}\left(t_{2}\right) \\
& \vdots \\
\left\langle U_{N_{s}}(x), \Phi_{k}(x)\right\rangle_{\Omega_{d}} & \approx \alpha_{k}\left(t_{N_{s}}\right) .
\end{aligned}
$$

Note that the temporal coefficients satisfy orthogonality properties over the discrete set $t \in\left\{t_{1}, t_{2}, \ldots, t_{N_{s}}\right\}$ :

$$
\sum_{i=1}^{N_{s}}\left\langle U_{i}(x), \Phi_{k}(x)\right\rangle_{\Omega_{d}}^{2} \approx \sum_{i=1}^{N_{s}} \alpha_{i}^{2}\left(t_{i}\right)=\lambda_{k} .
$$

For a more detailed discussion on the POD method, the reader is referred to Ravindran (2000), Ly and Tran (2001), Singh et al. (2001), Efe and Özbay (2003b, 2004) and the references therein.

Standing Assumption: The majority of works dealing with POD and model reduction applications presume that the flow is dominated by coherent modes and the quantities on the both sides of $u(x, t)$ and $\hat{u}(x, t)$ are indistinguishable, Ravindran (2000), Ly and Tran (2001), Singh et al. (2001), Efe and Özbay (2003a, b, 2004). Because of the dominance of coherent modes, the typical spread of the eigenvalues of the correlation matrix turns out to be logarithmic and the terms decay very rapidly in magnitude. This fact further enables us to assume that a reduced order representation, say with $M$ modes $\left(M \leq \min \left(R_{L}, N_{s}\right)\right)$ can also be written as an equality

$$
\hat{u}(x, t)=\sum_{i=1}^{M} \alpha_{i}(t) \Phi_{i}(x),
$$

and the reduced order model is derived under the assumption that (15) satisfies the governing PDE. Unsurprisingly, such an assumption results in a model having uncertainties. However, one should keep in mind that the goal is to find a model, which matches the infinite dimensional system in some sense of approximation with typically $M \ll R_{L} \leq N_{s}$. To assess how good such an expansion is, a percent energy measure is defined as follows

$$
E=100 \frac{\sum_{i=1}^{M} \lambda_{i}}{\sum_{i=1}^{R_{L}} \lambda_{i}},
$$

where the tendency of $E \rightarrow 100 \%$ means that the model captures the dynamical information contained in the snapshots well. Conversely, an insufficient model will be obtained if $E$ is far below $100 \%$. In the next section, we demonstrate how the boundary condition is transformed to an explicit control input in the ODEs.

\section{Model reduction with explicit control input}

In this section, we apply the POD technique to the Burgers equation described by (9) with $\epsilon=4$ as a known process parameter. The problem is specified with zero initial conditions, the homogeneous boundary condition at $x=0$ as $u(0, t)=0$ and Dirichlét type boundary condition at $x=1$ as $u(1, t)=\gamma(t)$. Since the POD scheme yields the decomposition in (9), according to the standing assumption, inserting $\hat{u}$ in the place of $u$ results in

$$
\begin{aligned}
\sum_{i=1}^{M} \dot{\alpha}_{i}(t) \Phi_{i}(x)= & \sum_{i=1}^{M} \alpha_{i}(t) \epsilon \frac{\partial^{2} \Phi_{i}(x)}{\partial x^{2}} \\
& -\left(\sum_{i=1}^{M} \sum_{j=1}^{M} \alpha_{i}(t) \alpha_{j}(t) \Phi_{i}(x) \frac{\partial \Phi_{j}(x)}{\partial x}\right) .
\end{aligned}
$$


Taking the inner product of both sides of (17) with $\Phi_{k}(x)$, which corresponds to the Galerkin projection, results in the equality in (18).

$$
\begin{aligned}
\dot{\alpha}_{k}(t)= & \sum_{i=1}^{M} \alpha_{i}(t) \epsilon\left\langle\Phi_{k}(x), \zeta_{i}(x)\right\rangle_{\Omega_{d}} \\
& -\left(\sum_{i=1}^{M} \sum_{j=1}^{M} \alpha_{i}(t) \alpha_{j}(t)\left\langle\Phi_{k}(x), \Phi_{i}(x) \tau_{j}(x)\right\rangle_{\Omega_{d}}\right),
\end{aligned}
$$

where $\zeta_{i}(x):=\partial^{2} \Phi_{i}(x) / \partial x^{2} \quad$ and $\quad \tau_{i}(x):=\partial \Phi_{i}(x) / \partial x$. As mentioned earlier, the effects of the external stimulus is implicit in the above equation. For this reason, choose the grid $\Omega_{d}^{\circ}:=\left\{x \mid x \in \bigcup_{i=0}^{S-2}(i \Delta x)\right\}$, where $S$ is the number of grid points considered for the numerical solution satisfying $(S-1) \Delta x=1$. Clearly, $\Omega_{d}^{\circ} \cup 1 \equiv \Omega_{d}$, or equivalently the boundary $\partial \Omega_{d}:=\{x \mid x=1\}$. According to these definitions, $\langle f(x), g(x)\rangle_{\Omega}=\langle f(\underline{x}), g(\underline{x})\rangle_{\Omega_{d}}=$ $\left(1 / N_{s}\right) f(\underline{x})^{\mathrm{T}} g(\underline{x})$, where the column vector $\underline{x}$ contains the elements of $\Omega_{d}$ in ascending order. In a similar fashion, one can define $\underline{x}^{\circ}$ as the column vector containing the elements of $\Omega_{d}^{\circ}$ in the same way. Taking the above partitioning into account, and rewriting (18) yield

$$
\begin{aligned}
N_{s} \dot{\alpha}_{k}(t)= & \sum_{i=1}^{M} \alpha_{i}(t) \epsilon \Phi_{k}^{\mathrm{T}}(\underline{x}) \zeta_{i}(\underline{x}) \\
& -\left(\sum_{i=1}^{M} \sum_{j=1}^{M} \alpha_{i}(t) \alpha_{j}(t) \Phi_{k}^{\mathrm{T}}(\underline{x})\left(\Phi_{i}(\underline{x}) \star \tau_{j}(\underline{x})\right)\right) \\
= & \sum_{i=1}^{M} \alpha_{i}(t) \epsilon \Phi_{k}^{\mathrm{T}}\left(\underline{x}^{\circ}\right) \zeta_{i}\left(\underline{x}^{\circ}\right)+\sum_{i=1}^{M} \alpha_{i}(t) \epsilon \Phi_{k}(1) \zeta_{i}(1) \\
& -\left(\sum_{i=1}^{M} \sum_{j=1}^{M} \alpha_{i}(t) \alpha_{j}(t) \Phi_{k}^{\mathrm{T}}\left(\underline{x}^{\circ}\right)\left(\Phi_{i}\left(\underline{x}^{\circ}\right) \star \tau_{j}\left(\underline{x}^{\circ}\right)\right)\right) \\
& -\left(\sum_{i=1}^{M} \sum_{j=1}^{M} \alpha_{i}(t) \alpha_{j}(t) \Phi_{k}(1) \Phi_{i}(1) \tau_{j}(1)\right),
\end{aligned}
$$

where $\star$ denotes the elementwise product operator.

Since the external inputs are not seen explicitly in (19), in what follows, the terms will be manipulated such that the two dynamics, namely the one enters directly with the boundary condition and the one governed by the PDE along the spatial direction are separated properly. The driving point is to notice that the solution in (15) must be satisfied at the boundaries as well. This gives the following information

$$
u(1, t)=\gamma(t)=\sum_{i=1}^{M} \alpha_{i}(t) \Phi_{i}(1) .
$$

Or $\alpha_{k}(t) \Phi_{k}(1)=\gamma(t)-\sum_{i=1}^{M}\left(1-\delta_{i k}\right) \alpha_{i}(t) \Phi_{i}(1)$. Inserting this into the second summation in (19) yields

$$
\begin{aligned}
\sum_{i=1}^{M} \alpha_{i}(t) \epsilon \Phi_{k}(1) \zeta_{i}(1)= & \alpha_{k}(t) \epsilon \Phi_{k}(1) \zeta_{k}(1) \\
& +\sum_{i=1}^{M}\left(1-\delta_{i k}\right) \alpha_{i}(t) \Phi_{k}(1) \zeta_{i}(1) \\
= & \gamma(t) \epsilon \zeta_{k}(1)+\sum_{i=1}^{M} \alpha_{i}(t) \epsilon\left(\Phi_{k}(1) \zeta_{i}(1)\right. \\
& \left.-\Phi_{i}(1) \zeta_{k}(1)\right) .
\end{aligned}
$$

Similarly, considering (20) for the last term of (19), we can perform the following rearrangement:

$$
\begin{aligned}
& \sum_{i=1}^{M} \sum_{j=1}^{M} \alpha_{i}(t) \alpha_{j}(t) \Phi_{k}(1) \Phi_{i}(1) \tau_{j}(1) \\
& \quad=\Phi_{k}(1) \sum_{i=1}^{M} \alpha_{i}(t) \Phi_{i}(1) \sum_{j=1}^{M} \alpha_{j}(t) \tau_{j}(1) \\
& \quad=\Phi_{k}(1) \gamma(t) \sum_{j=1}^{M} \alpha_{j}(t) \tau_{j}(1) .
\end{aligned}
$$

Summing up the all four terms of (19) results in

$$
\begin{aligned}
N_{s} \dot{\alpha}_{k}(t)= & \left(\sum_{i=1}^{M} \alpha_{i}(t) \epsilon \Phi_{k}^{\mathrm{T}}\left(\underline{x}^{\circ}\right) \zeta_{i}\left(\underline{x}^{\circ}\right)\right)+\gamma(t) \epsilon \zeta_{k}(1) \\
& +\sum_{i=1}^{M} \alpha_{i}(t) \epsilon\left(\Phi_{k}(1) \zeta_{i}(1)-\Phi_{i}(1) \zeta_{k}(1)\right) \\
& -\left(\sum_{i=1}^{M} \sum_{j=1}^{M} \alpha_{i}(t) \alpha_{j}(t) \Phi_{k}^{\mathrm{T}}\left(\underline{x}^{\circ}\right)\left(\Phi_{i}\left(\underline{x}^{\circ}\right) \star \tau_{j}\left(\underline{x}^{\circ}\right)\right)\right) \\
& -\left(\Phi_{k}(1) \gamma(t) \sum_{j=1}^{M} \alpha_{j}(t) \tau_{j}(1)\right) \\
= & \left(\sum_{i=1}^{M} \alpha_{i}(t) \epsilon\left(\Phi_{k}^{\mathrm{T}}(\underline{x}) \zeta_{i}\left(\underline{x}^{\prime}\right)-\Phi_{i}(1) \zeta_{k}(1)\right)\right) \\
& -\left(\sum_{i=1}^{M} \sum_{j=1}^{M} \alpha_{i}(t) \alpha_{j}(t) \Phi_{k}^{\mathrm{T}}\left(\underline{x}^{\circ}\right)\left(\Phi_{i}\left(\underline{x}^{\circ}\right) \star \tau_{j}\left(\underline{x}^{\circ}\right)\right)\right) \\
& +\left(\epsilon \zeta_{k}(1)-\Phi_{k}(1) \sum_{j=1}^{M} \alpha_{j}(t) \tau_{j}(1)\right) \gamma(t) .
\end{aligned}
$$

Defining the state vector as $\alpha=\left(\alpha_{1} \alpha_{2} \ldots \alpha_{M}\right)^{\mathrm{T}}$, it becomes obvious that the above model implies the following dynamical system for temporal components of the POD

$$
\dot{\alpha}=A \alpha-C(\alpha)+(B-D \alpha) \gamma
$$


where $A, D \in \Re^{M \times M}$ and $B, C \in \Re^{M}$. We have

$$
\begin{aligned}
& (A)_{k i}=\frac{1}{N_{s}} \epsilon\left(\Phi_{k}^{\mathrm{T}}(\underline{x}) \zeta_{i}(\underline{x})-\Phi_{i}(1) \zeta_{k}(1)\right), \\
& C(\alpha)=\left(\begin{array}{lll}
\alpha^{\mathrm{T}} C_{1} \alpha & \alpha^{\mathrm{T}} C_{2} \alpha \ldots \alpha^{\mathrm{T}} C_{M} \alpha
\end{array}\right)^{\mathrm{T}},
\end{aligned}
$$

where $\left(C_{k}\right)_{i j}=\left(1 / N_{s}\right) \Phi_{k}^{\mathrm{T}}\left(\underline{x}^{\circ}\right)\left(\Phi_{i}\left(\underline{x}^{\circ}\right) \star \tau_{j}\left(\underline{x}^{\circ}\right)\right)$.

$$
(B)_{k}=\frac{1}{N_{s}} \epsilon \zeta_{k}(1)
$$

and

$$
(D)_{k i}=\frac{1}{N_{s}} \tau_{i}(1) \Phi_{k}(1) .
$$

with $\alpha(0)=0$.

In the rest of this section, we discuss the important issues in modelling phase and demonstrate that the developed model performs well under the chosen conditions. In the model derivation stage, we set $\epsilon=4$ and collected the snapshots according to the following procedure: The end time is $1 \mathrm{sec}$ and time step is $1 \mathrm{msec}$, i.e. we have 1001 snapshots at each solution trial, and the $n$th trial is performed with $u(x, 0)=0$, $u(0, t)=0, u(1, t)=\gamma(t)=\sin (2 \pi(2 n-1 / 1.024) t)$, where $n=1,2, \ldots, 101$. Such a boundary excitation scheme covers frequencies approximately up to $200 \mathrm{~Hz}$. The snapshot collection scheme linearly samples 10 snapshots from each trial to build up the ensemble of snapshots, $\left\{U_{i}(x)\right\}_{i=1}^{N_{s}}$. During this procedure, due to its numerical stability and simplicity, Crank-Nicholson method is used as the numerical solver with $S=100$ spatial grid points i.e. $\Delta x=1 / 99$ (Farlow (1993) for details). Clearly, finer grids would be computationally intensive yet a solution obtained from a sparse grid may not yield a useful dynamic model. We set this resolution after a few trials of checking the left and right hand sides of the PDE in (9) numerically. Running a boundary regime described above excites reasonably large number of dynamical constituents of the Burgers system. In Efe and Özbay (2003b), the locality of the POD models has been emphasized and this problem is alleviated by maintaining the spectral diversity in the snapshots. In other words, executing the above described experiments gives us a model capturing those frequencies to some extent. Nevertheless, reliability of the resulting dynamical model should be viewed as a decreasing quantity as the operating conditions become dissimilar from the model generation conditions.

The simulations have shown that for $M<5$, the energy content is insufficient to rebuild the numerical data, on the other hand, for $M>7$, the basis functions are steeper and numerical differentiation errors become significant. For this reason, we set $M=5$, which captures $E=99.94 \%$ of the total energy. One should notice that the application of the above described procedure gives us the terms seen in (24) as well as the basis functions of the output equation in (10). Our expectation is to have a good match between the response to a test signal obtained from the numerical solver and from the dynamical system. For this purpose, we choose a signal given by (29) and illustrate the relevant subdomain of fast fourier transform (FFT) magnitude components in figure 1 . It should be visible that the chosen signal contains an admissible degrees of spectral richness to validate that the model works appropriately nearly below $200 \mathrm{~Hz}$. It has a distinguishable low frequency component to excite the diffusion term $\epsilon u_{x x}$, where the larger the $\epsilon$ the more diffusive the behaviour along the $x$-direction;

$$
\begin{aligned}
& \gamma(t)=\sin (2 \pi 30(1-t) t)+\operatorname{sign}(\sin (2 \pi 10 t)) \\
& \text { for } 0 \leq t \leq 1.023 \mathrm{sec} .
\end{aligned}
$$

Figure 2 illustrates the results for each individual mode. It is visible from the figure that the low frequency components are well reconstructed, while the sharp transitions are smoothed due to the underlying philosophy of the POD scheme, which is based on the truncation of high frequency components.

A combined view is presented in figure 3 . Both the numerical solution and the reconstructed solution are plotted and it is observed that the reduced order model is a fairly good representative of the infinite dimensional system under the conditions illustrated.

\section{Connections between the reduced order model and reduced order observer}

In this section, we discuss the implications of model reduction in terms of target dynamics and the observer. Since the basis set $\Phi(x)=\left\{\Phi_{1}(x), \Phi_{2}(x), \ldots, \Phi_{R_{L}}(x)\right\}$ describes the way in which the variables must spread over $\Omega$, it is natural to assume that a reduced order model of the observer could admit the same basis set. This would unsurprisingly result in a dynamical model of the form

$$
\begin{gathered}
\dot{\beta}(t)=A \beta(t)-C(\beta(t))+(B-D \beta(t)) \gamma(t) \\
+K \frac{\Psi\left(x_{m}\right)^{\mathrm{T}}}{\Psi\left(x_{m}\right)^{\mathrm{T}} \Psi\left(x_{m}\right)}(\alpha(t)-\beta(t)), \\
\hat{v}(x, t)=\sum_{i=1}^{M} \Phi_{i}(x) \beta_{i}(t),
\end{gathered}
$$

where, $x_{m}$ is a measurement location to observe $\hat{v}$ and $\Psi\left(x_{m}\right):=\left(\Phi_{1}\left(x_{m}\right) \Phi_{2}\left(x_{m}\right) \ldots \Phi_{M}\left(x_{m}\right)\right)^{\mathrm{T}}$. 


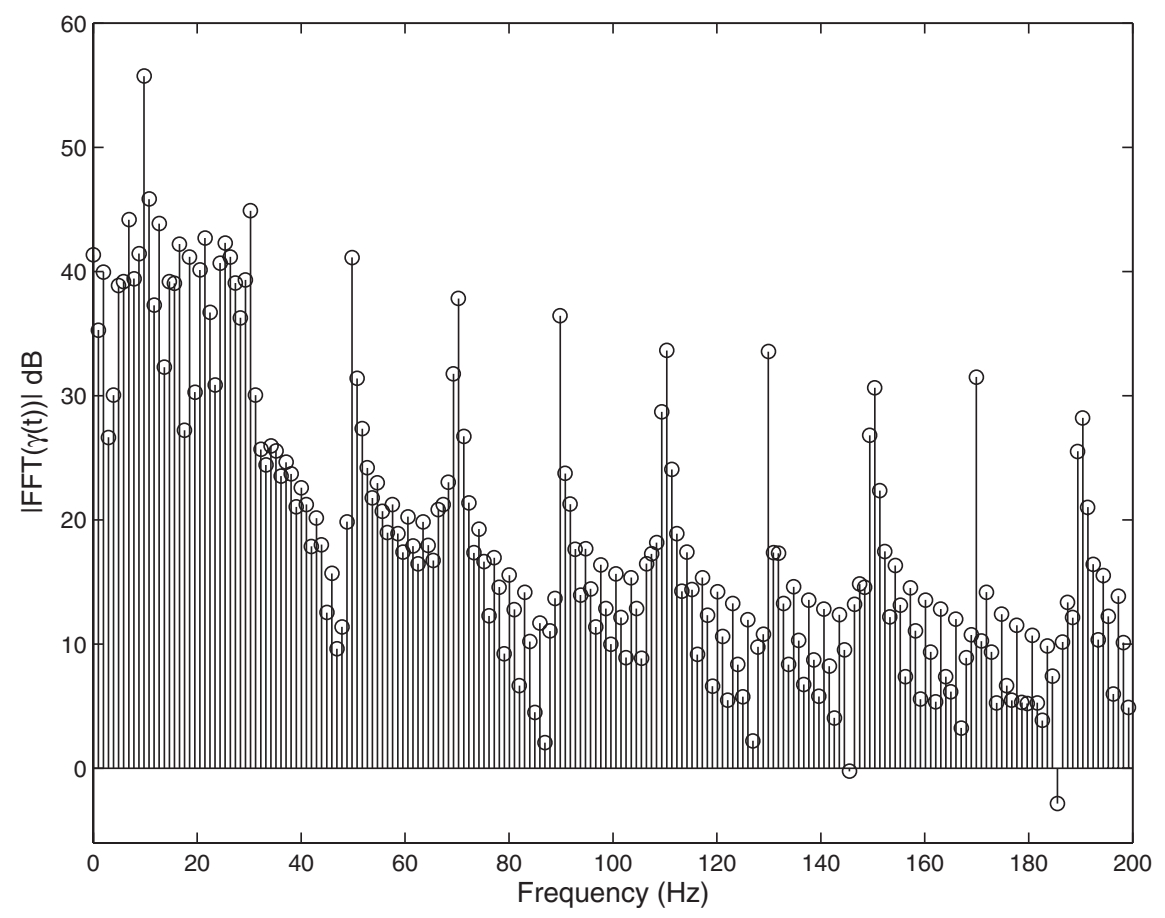

Figure 1. FFT magnitude view of the $\gamma(t)$ for frequencies below $200 \mathrm{~Hz}$.

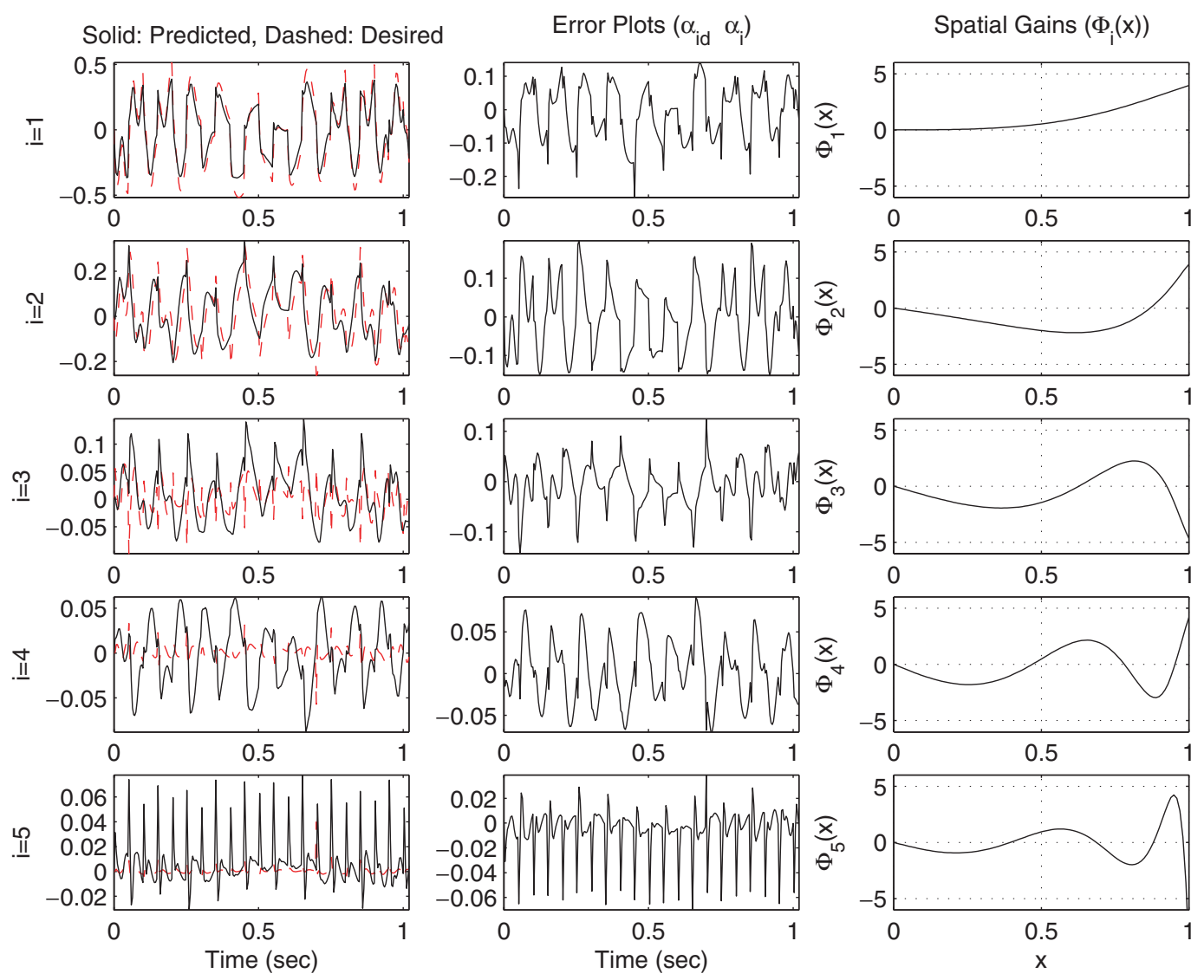

Figure 2. Obtained temporal information and the basis set. 

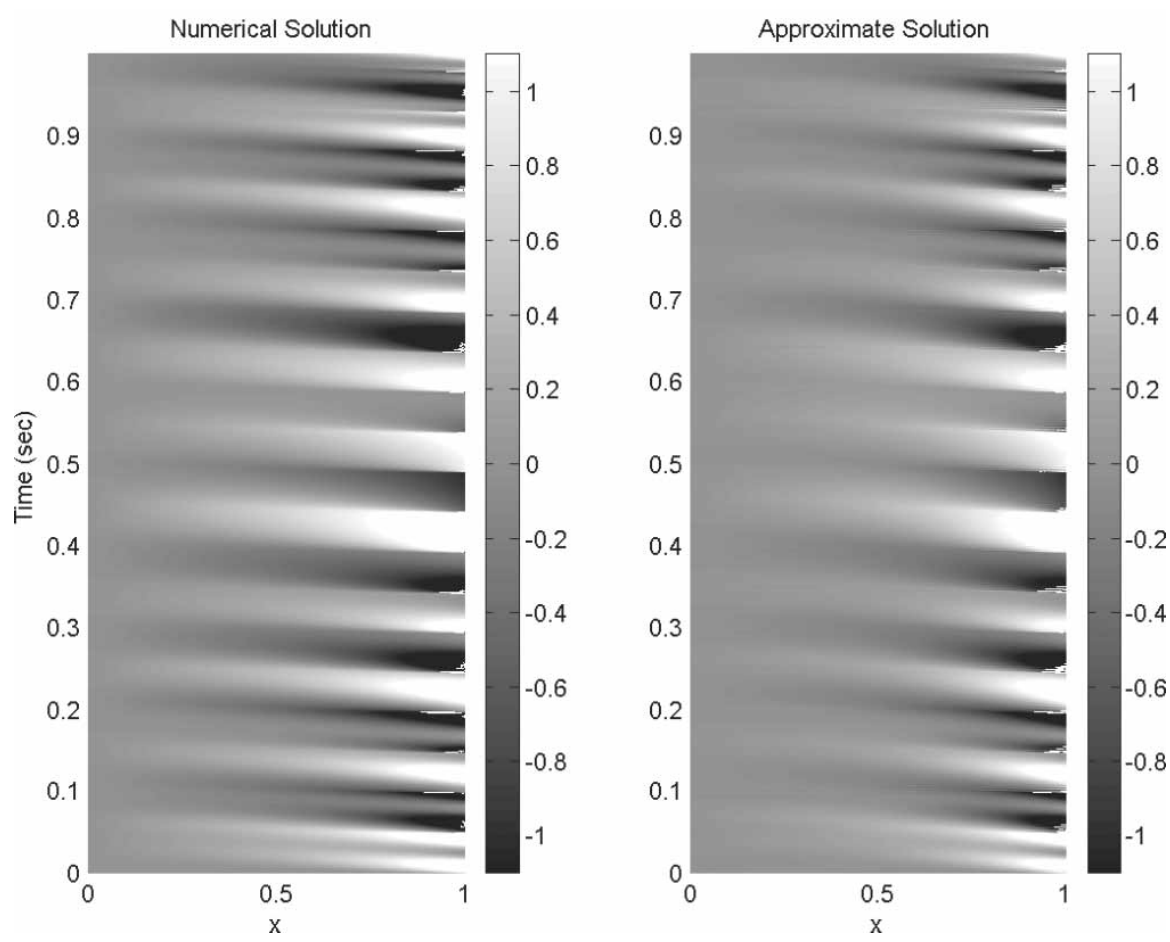

Figure 3. Numerical and approximate solutions.

Remark 1: Although the goal is to estimate the set of representative state information of the PDE system, the observer in (30) is designed to reconstruct the states $(\alpha)$ of the reduced order representation of the target system, i.e. $\beta(t) \rightarrow \alpha(t)$ as $t \rightarrow \infty$. For this reason, the modified form of (30), which is given below, becomes the equivalent and physically implementable form of the observer,

$$
\begin{aligned}
\dot{\beta}(t)= & A \beta(t)-C(\beta(t))+(B-D \beta(t)) \gamma(t) \\
& +K^{\prime}\left(u\left(x_{m}, t\right)-\hat{v}\left(x_{m}, t\right)\right),
\end{aligned}
$$

where $K^{\prime}=K /\left(\Psi\left(x_{m}\right)^{\mathrm{T}} \Psi\left(x_{m}\right)\right)$. Whether or not this is reasonable is absolutely dependent upon the standing assumption given at the end of $\S 3$. The observer in (32) reads the feedback from $x=x_{m}$, and it therefore forces $\hat{v}\left(x_{m}, t\right) \rightarrow u\left(x_{m}, t\right) \quad$ by rebuilding the state information progressively.

Remark 2: Let $e_{i}(t):=\alpha_{i}(t)-\beta_{i}(t) \quad$ and $\quad e(t):=$ $\left(e_{1}(t) e_{2}(t) \ldots e_{M}(t)\right)^{\mathrm{T}}$. Use of an observer of the form (31)-(32) forces the following equivalence in between the Lyapunov function in (2) and the one derived below

$$
\begin{aligned}
\hat{V}_{o} & =\frac{1}{2} \int_{0}^{1}(\hat{u}(x, t)-\hat{v}(x, t))^{2} \mathrm{~d} x \\
& =\frac{1}{2} \int_{0}^{1}\left(\sum_{i=1}^{M} \alpha_{i}(t) \Phi_{i}(x)-\sum_{i=1}^{M} \beta_{i}(t) \Phi_{i}(x)\right)^{2} \mathrm{~d} x
\end{aligned}
$$

$$
\begin{aligned}
= & \frac{1}{2} \int_{0}^{1}\left(\sum_{i=1}^{M}\left(\alpha_{i}(t)-\beta_{i}(t)\right) \Phi_{i}(x)\right)^{2} \mathrm{~d} x \\
= & \frac{1}{2} \int_{0}^{1}\left(\sum_{i=1}^{M} e_{i}(t) \Phi_{i}(x)\right)^{2} \mathrm{~d} x \\
= & \frac{1}{2} \int_{0}^{1}\left(\sum_{i=1}^{M} e_{i}(t)^{2} \Phi_{i}(x)^{2}\right) \mathrm{d} x \\
& +\int_{0}^{1}\left(\sum_{i=1}^{M} \sum_{j=1, j \neq i}^{M} e_{i}(t) e_{j}(t) \Phi_{i}(x) \Phi_{j}(x)\right) \mathrm{d} x \\
= & \frac{1}{2} \sum_{i=1}^{M}\left(e_{i}(t)^{2} \int_{0}^{1} \Phi_{i}(x)^{2} \mathrm{~d} x\right) \\
& +\sum_{i=1}^{M} \sum_{j=1, j \neq i}^{M}\left(e_{i}(t) e_{j}(t) \int_{0}^{1} \Phi_{i}(x) \Phi_{j}(x) \mathrm{d} x\right) \\
= & \frac{1}{2} \sum_{i=1}^{M}\left(e_{i}(t)^{2} \delta_{i i}\right)+\sum_{i=1}^{M} \sum_{j=1, j \neq i}^{M}\left(e_{i}(t) e_{j}(t) \delta_{i j}\right) \\
= & \frac{1}{2} \sum_{i=1}^{M} e_{i}(t)^{2}=\frac{1}{2} e(t)^{\mathrm{T}} e(t) .
\end{aligned}
$$

The above result is in good compliance with the Lyapunov theory and the tools of standard design techniques. Ensuring the negative definiteness of $\hat{V}_{o}$ is equivalent to asymptotically stable reconstruction of the state information in $\mathfrak{R}^{M}$. The connection between 
$\hat{V}_{o}$ and the infinite dimensional version in (2) in value is tightly dependent upon the energy context and the extent to which the standing assumption is fulfilled (refer to §2). In summary, the better the reduced order model describes the original dynamics, the better the reduced order observer estimates the dynamically significant states of the infinite dimensional system.

\section{A simulation study}

In this section, we discuss a set of simulation results based on the modified form of the observer in (31)-(32). That is to say, the feedback is read from the infinite dimensional system and the observer is low dimensional and $K^{\prime}=10$. Clearly, this one is the realistic implementation of the observation mechanism, yet the evidence of reconstruction performance is indirect. Technically speaking, an infinite dimensional observer is shown to be useful for infinite dimensional plant in the Lyapunov sense. Similarly, the implications of this in $\Re^{M}$ is shown to be compatible with the Lyapunov stability conclusions. A natural expectation of such a pattern is the usefulness of a low dimensional model for estimating the dominant states of the infinite dimensional system in the sense of POD based order reduction.

Having these in mind, we choose a boundary regime as shown in the top row of figure 4 . The signal is a noise corrupted one and it has a sinusoidal component during the first $0.5 \mathrm{sec}$ time, and a train of pulses during the rest of the simulation. The noiseless signal runs in between \pm 1 . This signal is used to excite both the PDE and the low dimensional observer. In order to make the observer design more reasonable, further two noise sequences, which are band limited white noises and are of power $1 \mathrm{e}-5$, are injected onto the input signal (boundary condition) of the PDE and the measured response from the PDE. Such an excitation strategy lets us have the excitation of many modes of the PDE and the low dimensional model, and causes some amount of uncertainty making it a challenge to extract a descriptive state information.

In order to illustrate the performance of the observer, one needs to test it for a reasonably rich set of physical locations from $\Omega_{d}$. For this reason, a time varying measurement location is set, i.e. we have $x_{m}(t)$ as shown in the bottom row of figure 4 . The above described boundary and measurement conditions constitute a good selection to test the reconstruction performance of the proposed observer.
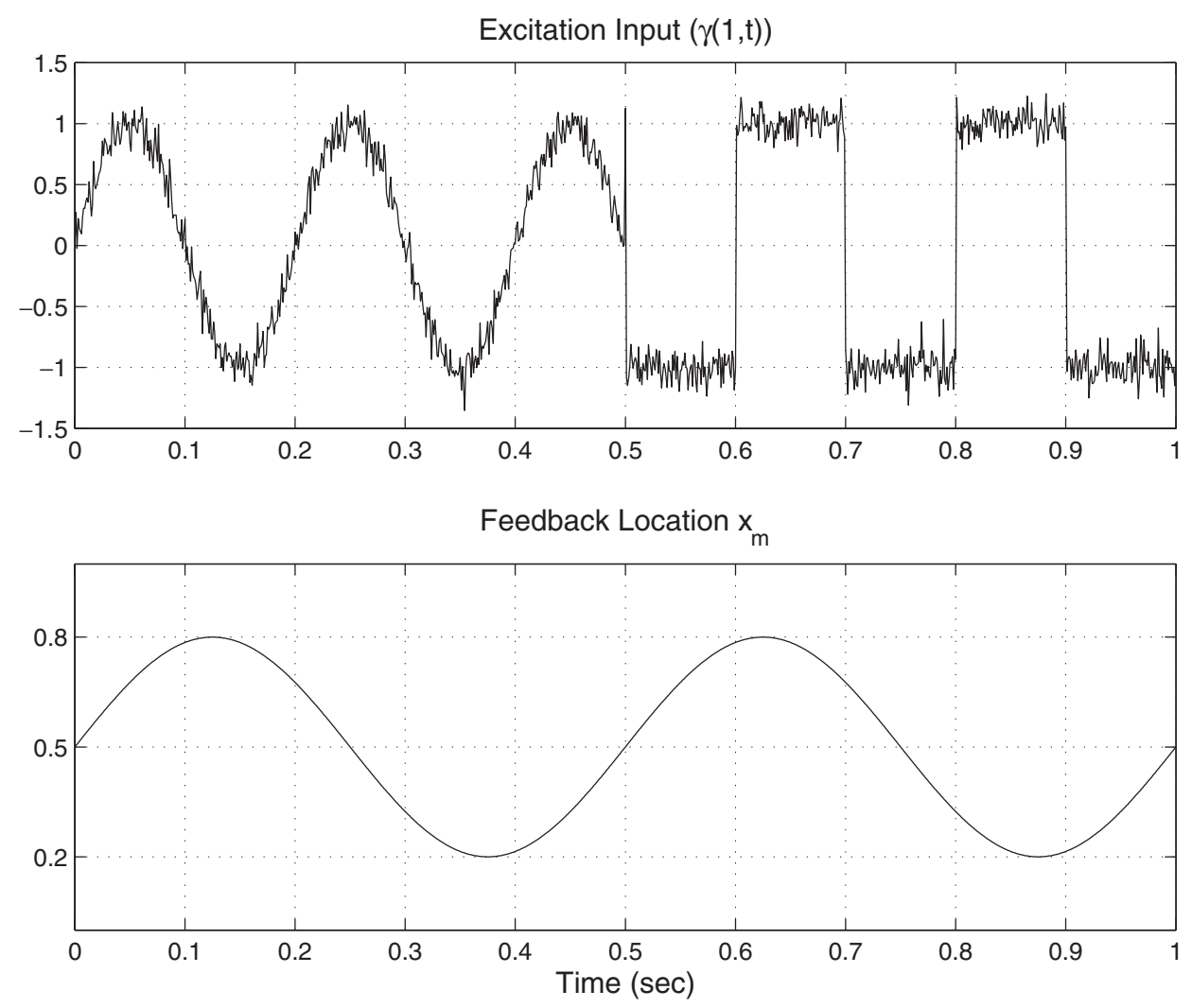

Figure 4. Applied boundary excitation $(\gamma(1, t))$ and the behaviour in moving feedback location $\left(x_{m}\right)$. 

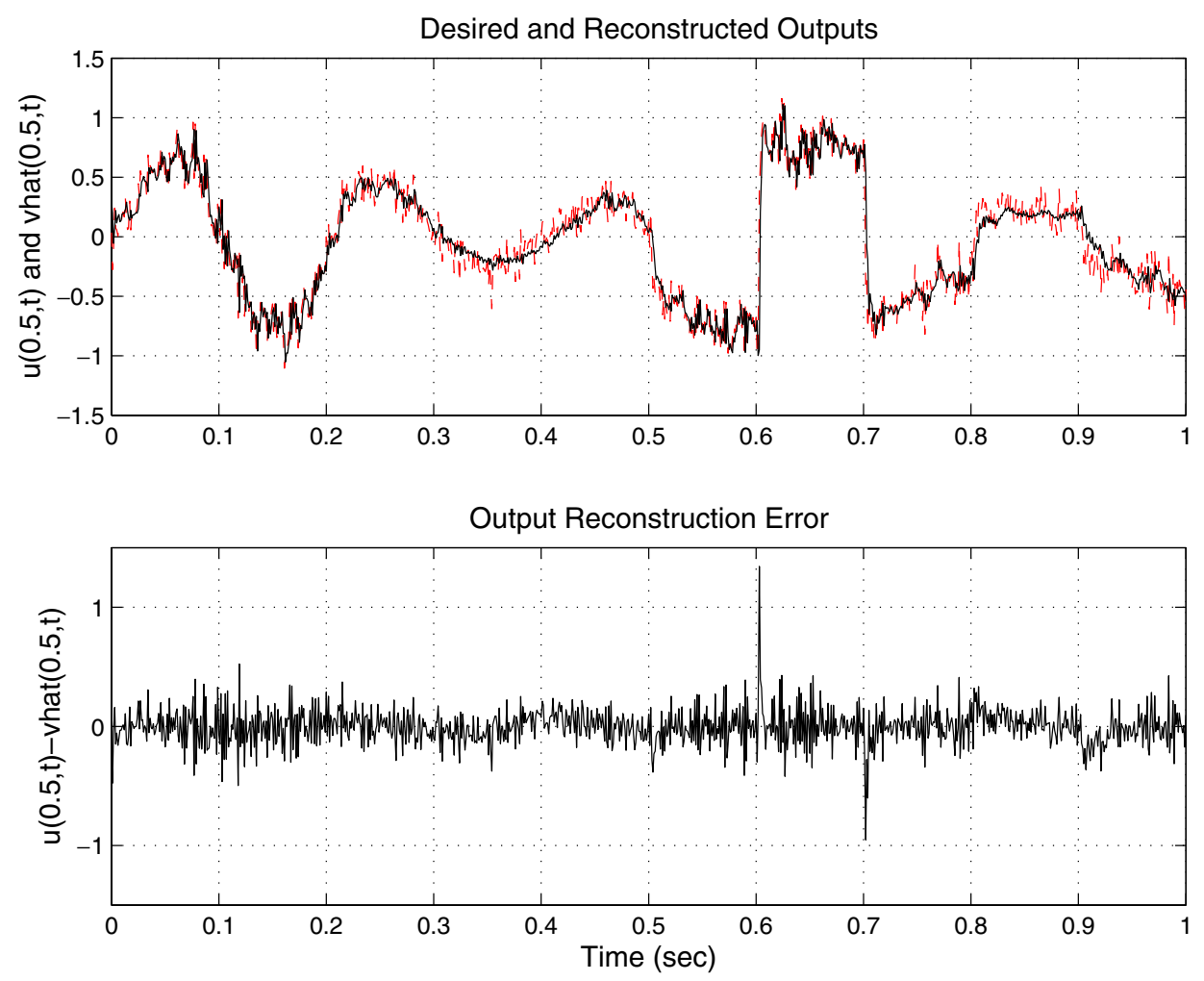

Figure 5. Desired and the reconstructed outputs, and the discrepancy between them.

In the top row of figure 5, two curves are plotted together. The one obtained from the PDE is depicted as a dashed line while the output of the observer is shown as a solid line. It is clearly distinguishable that the observer output is very close to the system output specified at the physical location $x_{m}(t)$. The difference between these two curves is plotted in figure 5. Two facts should be noticed from this figure: Firstly, the error trend is very close to zero during the first $0.5 \mathrm{sec}$ time. This means that if the signal to be followed is smooth then the mechanism accurately rebuilds the target. Secondly, the remaining $0.5 \mathrm{sec}$ time of the simulation indicates that the sharp changes in the signal being tracked causes expectedly large fluctuations, but these are convergent. These two facts make the indirect evidence of quick and accurate reconstruction very clear.

A last remark in this section is on the state information. A designer pursuing the design of a control system would find significantly important information within the presented mechanism. From this point of view, the possibility of reaching the dominant states of an infinite dimensional system would let us design feedback control mechanisms for PDE systems, which do not directly enjoy the strength of the well-established finite dimensional control systems theory.

\section{Relationship to flow modelling}

The current paper presents the results obtained through the course of a larger research project on areodynamic flow control. The considered problem and its extensions are accurately described in Debiasi and Samimy (2003), Samimy et al. (2003) and Yan et al. (2004). Briefly, the system is a rectangular cavity equipped with a set of sensor/actuator and host computing periphery. The regimes characterized by Mach 0.25 through 0.5 are considered as there are single mode and multi mode resonances making it a challenge to capture the pressure fluctuations observed from the process. The model development has greatly benefited from the POD technique (Caraballo et al. 2004) yet the models obtained for the Navier-Stokes equations need some further improvement to match the desired profiles better. The results presented in the cited references and those in the current paper are in good compliance in the sense that POD is a very powerful alternative in extracting the dynamical composition of spatially continuous systems. The future work of the authors is therefore to focus on developing an observer for the relevant form of the Navier-Stokes equations and utilize it within a closed loop control system. Regarding the technical details, such as the sensors, actuators, digital signal processing units and the computing devices, one should 
refer to Debiasi and Samimy (2003), Samimy et al. (2003) and Yan et al. (2004).

\section{Conclusions}

This paper discusses the design and implementation issues for reduced order and infinite dimensional forms of observers of Burgers equation. The infinite dimensionality and nonlinearity of the equation, which are similar to those in Navier-Stokes equations, are the motivating factors. POD is used to decompose the solution into characterizing modes and a dynamical model containing the control input is built by manipulating the expansion appropriately.

The paper demonstrates that an infinite dimensional observer can lead to the negative definiteness of a suitably defined Lyapunov function, and the same relation could be observed in the finite dimensional space for reduced order models. In the simulations, the finite dimensional observer is used with the infinite dimensional system to illustrate the efficacy of the proposed observer dynamics. The relevance of the presented work and the real-time counterpart are discussed from the point of POD use as a modelling tool. The results are in good compliance with the theoretical foundations, and this advances the subject area to the possibility of existence of such an observer for more complicated systems, such as flows governed by Navier-Stokes equations.

\section{Acknowledgments}

This work was supported in part by AFRL/VA and AFOSR under contract no F33615-01-2-3154.

The authors would like to thank Dr. J.H. Myatt, Dr. J. DeBonis, Dr. M. Debiasi, Dr. R.C. Camphouse, Dr. P. Yan, X. Yuan and E. Caraballo for fruitful discussions in devising the presented work.

\section{References}

J.A. Burns, B.B. King and L. Zietsman, "On the computation of singular functional gains for linear quadratic optimal boundary control problems", in Proceedings of the 3rd Theoretical Fluid Mechanics Meeting, St. Louis, AIAA 2002-3074, June 24-26, 2002a.
J.A. Burns, B.B. King, A.D. Rubio and L. Zietsman, "Functional gain computations for feedback control of a thermal fluid", in Proceedings of the 3rd Theoretical Fluid Mechanics Meeting, St. Louis, AIAA 2002-2992, June 24-26, 2002 b.

E. Caraballo, J. Malone, M. Samimy and J. Debonis, "A study of subsonic cavity flows: low dimensional modelling", AIAA Paper 2004-2124, 2004.

M. Debiasi and M. Samimy, "An experimental study of the cavity flow for closed-loop flow control”, AIAA Paper 2003-4003, June 2003.

M.Ö. Efe and H. ÖZbay, "Integral action based Dirichlét boundary control of Burgers equation", in IEEE International Conference on Control Applications (CCA'2003), Istanbul, Turkey, June 23-25, 2003a, pp.1267-1272.

M.Ö. Efe and H. ÖZbay, "Proper orthogonal decomposition for reduced order modelling: 2D heat flow", in IEEE International Conference on Control Applications (CCA'2003), Istanbul, Turkey, June 23-25, 2003b, pp. 1273-1278.

M.Ö. Efe and H. Özbay, "Low dimensional modelling and Dirichlét boundary bontroller design for Burgers equation", International Journal of Control, 77(10), pp. 895-906, 2004.

S.J. Farlow, Partial Differential Equations for Scientists and Engineers, New York: Dover Publications Inc., 1993, pp. 317-322.

S. Gügercin and A.C. Antoulas, "A comparative study of 7 algorithms for model reduction", in Proceedings of the 39th IEEE Conference on Decision and Control, Sydney, Australia, Dec. $12-15,2000$.

M. Hinze and S. Volkwein, "Analysis of instantaneous control for Burgers equation", Nonlinear Analysis, 50, pp. 1-26, 2002.

M. Krstić, "On global stabilization of Burgers' equation by boundary control", Systems and Control Letters, 37, pp. 123-141, 1999.

W.-J. Liu and M. Krstić, "Backstepping boundary control of Burgers' equation with actuator dynamics", Systems and Control Letters, 41, pp. 291-303, 2000.

W.-J. Liu and M. Krstić, “Adaptive control of Burgers' equation with unknown viscosity", International Journal of Adaptive Control and Signal Processing, 15, pp. 745-766, 2001.

H.V. Ly and H.T. Tran, "Modeling and control of physical processes using proper orthogonal decomposition", Mathematical and Computer Modelling of Dynamical Systems, 33, pp. 223-236, 2001.

H.M. Park and Y.D. Jang, "Control of Burgers equation by means of mode reduction", International Journal of Engineering Science, 38, pp. 785-805, 2002.

S.S. Ravindran, "A reduced order approach for optimal control of fluids using proper orthogonal decomposition", International Journal for Numerical Methods in Fluids, 34, pp. 425-488, 2000.

M. Samimy, M. Debiasi, E. Caraballo, H. Özbay, M.Ö. Efe, X. Yuan, J. Debonis and J.H. Myatt, "Development of closed-loop flow control for cavity flows", AIAA Paper 2003-4258, 2003.

S.N. Singh, J.H. Myatt, G.A. Addington, S. Banda and J.K. Hall, "Optimal feedback control of vortex shedding using proper orthogonal decomposition models", Transactions of the ASME: Journal of Fluids Eng., 123, pp. 612-618, 2001.

R. Vedantham, "Optimal control of the viscous Burgers equation using an equivalent index method", Journal of Global Optimization, 18, pp. 255-263, 2000.

P. Yan, M. Debiasi, X. Yuan, E. Carabalo, M.Ö. Efe, H. Özbay, M. Samimy, J. Debonis, R.C. Camphouse, J.H. Myatt, A. Serrani and J. Malone, "Controller design for active closed-loop control of cavity flows", AIAA Paper 2004-0573, 2004. 